\title{
Octahedral and Tetrahedral Coinage Metal Clusters: Is Three-dimensional d-orbital Aromaticity Viable?
}

Clémence Corminboeuf, Chaitanya S. Wannere, ${ }^{*}$ Debjani Roy, R. Bruce King, Paul v. R. Schleyer*

Center for Computational Chemistry and Department of Chemistry, University of Georgia, Athens, Georgia 30602.

Complete reference 19: M. J. Frisch, M. J.; Trucks, G. W.; Schlegel, H. B.; Scuseria, G. E.; Robb, M. A.; Cheeseman, J. R.; Montgomery, Jr. J. A.; Vreven, T.; Kudin, K. N.;

Burant, J. C.; Millam, J. M.; Iyengar, S. S.; Tomasi, J.; Barone, V.; Mennucci, B.; Cossi, M.; Scalmani, G.; Rega, N.; Petersson, G. A.; Nakatsuji, H.; Hada, M.; Ehara, M.;

Toyota, K.; Fukuda, R.; Hasegawa, J.; Ishida, M.; Nakajima, T.; Honda, Y.; Kitao, O.; Nakai, H.; Klene, M.; Li, X.; Knox, J. E.; Hratchian, H. P.; Cross, J. B.; Adamo, C.; Jaramillo, J.; Gomperts, R.; Stratmann, R. E.; Yazyev, O.; Austin, A. J.; Cammi, R.; Pomelli, C.; Ochterski, J. W.; Ayala, P. Y.; Morokuma, K.; Voth, G. A.; Salvador, P.; Dannenberg, J. J.; Zakrzewski, V. G.; Dapprich, S.; Daniels, A. D.; Strain, M. C.; Farkas, O.; Malick, D. K.; Rabuck, A. D.; Raghavachari, K.; Foresman, J. B.; Ortiz, J. V.; Cui, Q.; Baboul, A. G.; Clifford, S.; Cioslowski, J.; Stefanov, B. B.; Liu, G.; Liashenko, A.; Piskorz, P.; Komaromi, I.; Martin, R. L.; Fox, D. J.; Keith, T.; Al-Laham, M. A.; Peng, C. Y.; Nanayakkara, A.; Challacombe, M.; Gill, P. M. W.; Johnson, B.; Chen, W.; Wong, M. W.; Gonzalez, C.; Pople, J. A. Gaussian 03, revision C.02 ; Gaussian, Inc.:

Wallingford, CT, 2004. 
Table A Cartesian co-ordinates of all the structures:

\section{$\mathrm{Cu}_{4} \mathrm{Li}_{4}$ inner $\mathrm{Li}_{4}$ tetrahedron:}

$1 \backslash 1 \backslash$ GINC-UGA-PVRS-

PC28PVRS\FOpt\RPW91PW91 \LANL2DZICu4Li4IWANNERE\22-F

eb-2005\0\\# RPW91PW91/LANL2DZ OPT FREQ=NORAMAN POP=NONE

NAME$=W A N N E R E I$

ICu4Li4 Td symmetry Li4 Td cage //PW91/LANL2DZII0,1\Li,1.033230101,1.0

33230101,1.033230101\Li,-1.033230101,-1.033230101,1.033230101\Li,-1.03

3230101,1.033230101,-1.033230101\Li,1.033230101,-1.033230101,-1.033230

$101 \backslash \mathrm{Cu},-1.4570089013,-1.4570089013,-1.4570089013 \backslash \mathrm{Cu}, 1.4570089013,1.457$

0089013,-1.4570089013\Cu,1.4570089013,-1.4570089013,1.4570089013\Cu,-1

$.4570089013,1.4570089013,1.4570089013 \backslash \backslash$ Version=x86-Linux-G98RevA.5\HF=

-815.1070892\RMSD=8.077e-09\RMSF=1.605e-05\PG=TD [4C3(Cu1.Li1)]\\@

\section{$\mathrm{Cu}_{4} \mathrm{Li}_{4}$ inner $\mathrm{Cu}_{4}$ tetrahedron with $\mathrm{Li}$ capping:}

$1 \backslash 1 \backslash$ GINC-UGA-PVRS-

PC31PVRS\FOpt\RPW91PW91 \LANL2DZ\Cu4Li4IWANNERE\12-F

eb-2005\0\\# PW91PW91/LANL2DZ OPT FREQ=NORAMAN POP=NONE

NAME$=$ WANNEREII

Cu4Li4 Td symmetry Schleyer molecule //PW91/LANL2DZII0,1\Cu,0.89026863

$24,0.8902686324,0.8902686324 \backslash \mathrm{Cu},-0.8902686324,-0.8902686324,0.89026863$

$24 \mathrm{lCu},-0.8902686324,0.8902686324,-0.8902686324 \mathrm{ICu}, 0.8902686324,-0.8902$

$686324,-0.8902686324 \backslash \mathrm{Li},-1.5205958252,-1.5205958252,-1.5205958252 \backslash \mathrm{Li}, 1$

$.5205958252,1.5205958252,-1.5205958252 \backslash \mathrm{Li}, 1.5205958252,-1.5205958252,1$

$.5205958252 \backslash \mathrm{Li},-1.5205958252,1.5205958252,1.5205958252 / \backslash V \operatorname{Version}=x 86-\mathrm{Li}$

nux-G98RevA.5\HF=-815.1296397\RMSD=7.126e-09\RMSF=1.140e-04\PG=TD [4C3

(Li1.Cu1)]\@

$\mathrm{Ag}_{4} \mathrm{Li}_{4}$ inner $\mathrm{Li}_{4}$ tetrahedron:

$1 \backslash 1 \backslash$ GINC-UGA-PVRS-

PC34PVRSIFOpt\RPW91PW91 LLANL2DZ\Ag4Li4IWANNEREI02-M ay-2005\0\\# PW91PW91/LANL2DZ OPT FREQ=NORAMAN POP=NONE

NAME $=$ WANNERE $S$

CFCYC=300 GUESS=READ GEOM=ALLCHECK\\Ag4Li4 Td symmetry //PW91/LANL2DZI

10,1\Li,1.0563997763,1.0563997763,1.0563997763\Li,-1.0563997763,-1.056

3997763,1.0563997763\Li,-1.0563997763,1.0563997763,-1.0563997763\Li,1.

0563997763,-1.0563997763,-1.0563997763\Ag,-1.5305323697,-1.5305323697, $-1.5305323697 \backslash \mathrm{Ag}, 1.5305323697,1.5305323697,-1.5305323697 \backslash \mathrm{Ag}, 1.53053236$

$97,-1.5305323697,1.5305323697 \backslash \mathrm{Ag},-1.5305323697,1.5305323697,1.53053236$

97\IVersion=x86-Linux-G98RevA.5 $\backslash \mathrm{HF}=-613.4673018 \backslash \mathrm{RMSD}=6.772 \mathrm{e}-09 \backslash \mathrm{RMSF}=1$. 443e-04\PG=TD [4C3(Ag1.Li1)]\\ 


\section{$\mathrm{Ag}_{4} \mathrm{Li}_{4}$ inner $\mathrm{Ag}_{4}$ tetrahedron with $\mathrm{Li}$ capping:}

$1 \backslash 1 \backslash$ GINC-UGA-PVRS-

PC32PVRS\FOpt\RPW91PW91\LANL2DZ\Ag4Li4\WANNERE $\backslash 19-F$

eb-2005\0\\# RPW91PW91/LANL2DZ OPT FREQ=NORAMAN POP=NONE

NAME $=$ WANNERE

$\backslash$ Au4Li4 Td symmetry (Ag4 Td cluster) //PW91/LANL2DZI10,1\Ag,1.05196171 07,1.0519617107,1.0519617107\Ag,-1.0519617107,-1.0519617107,1.05196171

07\Ag,-1.0519617107,1.0519617107,-1.0519617107\Ag,1.0519617107,-1.0519

617107,-1.0519617107\Li,-1.5177438582,-1.5177438582,-1.5177438582\Li, 1

$.5177438582,1.5177438582,-1.5177438582 \backslash \mathrm{Li}, 1.5177438582,-1.5177438582,1$

$.5177438582 \backslash \mathrm{Li},-1.5177438582,1.5177438582,1.5177438582 \backslash 1 V \operatorname{Version}=x 86-\mathrm{Li}$

nux-G98RevA.5\HF=-613.4437829\RMSD=9.664e-09\RMSF=6.015e-06\PG=TD [4C3 (Li1.Ag1)]川@

\section{$\mathrm{Au}_{4} \mathrm{Li}_{4}$ inner $\mathrm{Li}_{4}$ tetrahedron:}

$1 \backslash 1 \backslash$ GINC-UGA-PVRS-

PC28PVRS \FOpt\RPW91PW91 \LANL2DZ\Au4Li4IWANNEREI09-M

ay-2005\0\\# PW91PW91/LANL2DZ OPT POP=NONE NAME=WANNERE\\Au4Li4 Td sym metry //PW91/LANL2DZl $10,1 \mathrm{Li}, 1.0719302232,1.0719302232,1.0719302232 \mathrm{Li}$ $,-1.0719302232,-1.0719302232,1.0719302232 \backslash \mathrm{Li},-1.0719302232,1.071930223$

2,-1.0719302232\Li,1.0719302232,-1.0719302232,-1.0719302232\Au,-1.4611 850309,-1.4611850309,-1.4611850309\Au,1.4611850309,1.4611850309,-1.461 $1850309 \backslash \mathrm{Au}, 1.4611850309,-1.4611850309,1.4611850309 \backslash \mathrm{Au},-1.4611850309,1$. 4611850309,1.4611850309\IVersion=x86-Linux-G98RevA.5\HF=-572.4557916\R $\mathrm{MSD}=5.317 \mathrm{e}-09 \backslash \mathrm{RMSF}=1.268 \mathrm{e}-06 \backslash \mathrm{PG}=\mathrm{TD}$ [4C3(Au1.Li1)]\@

\section{$\mathrm{Cu}_{6} \mathrm{Li}_{2} C_{2 v}$ isomer:}

$1 \backslash 1 \backslash$ GINC-UGA-PVRS-

PC34PVRSIFOpt\RPW91PW91 \LANL2DZICu6Li2IWANNEREI29-N ov-2004l0\॥ RPW91PW91/LANL2DZ OPT FREQ=NORAMAN POP=NONE $\mathrm{NAME}=\mathrm{WANNERE}$

SCFCYC=300\\Cu6Li2 C2v singlet //PW91PW91/LANL2DZII0,1\Cu,-1.225134731 $8,-0.0604327246,1.2741078527 \backslash \mathrm{Cu}, 1.2251347318,-0.0604327246,1.274107852$

$7 \backslash \mathrm{Cu}, 1.2251347318,-0.0604327246,-1.2741078527 \backslash \mathrm{Cu},-1.2251347318,-0.0604$ $327246,-1.2741078527 \mathrm{Cu}, 0 ., 1.65268126,0.1 \mathrm{Cu}, 0 .,-1.7964708268,0 . \mathrm{Li},-2$. $5370910874,1.8633489153,0.1 \mathrm{Li}, 2.5370910874,1.8633489153,0.11$ Version $=x 8$

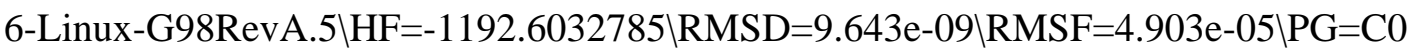
2V [C2(Cu1Cu1),SGV(Li2),X(Cu4)]\@

\section{$\mathrm{Cu}_{6} \mathrm{Li}_{2} C_{2 h}$ isomer:}

$1 \backslash 1 \backslash$ GINC-UGA-PVRS-

PC30PVRS \FOpt\RPW91PW91\LANL2DZICu6Li2\WANNERE\24-N 
ov-2004\0\\\# RPW91PW91/LANL2DZ OPT FREQ=NORAMAN POP=NONE NAME $=$ WANNERE

SCFCYC=300\\Cu6Li2 C2h symmetry \0,1\Cu,-0.7999004471,-1.2780559664,0. $9188379442 \backslash \mathrm{Cu},-0.7999004471,1.2780559664,0.9188379442 \backslash \mathrm{Cu}, 0.7999004471$, $-1.2780559664,-0.9188379442 \backslash \mathrm{Cu}, 0.7999004471,1.2780559664,-0.9188379442$ $\backslash \mathrm{Cu}, 1.402151408,0 ., 1.0615003797 \backslash \mathrm{Cu},-1.402151408,0 .,-1.0615003797 \backslash \mathrm{Li}, 0$. 2069450201,0.,-3.0791944578\Li,-0.2069450201,0.,3.0791944578।IVersion= x86-Linux-G98RevA.5\HF=-1192.5970798\RMSD=6.015e-09\RMSF=6.240e-05\PG= $\mathrm{C} 02 \mathrm{H}[\mathrm{SGH}(\mathrm{Cu} 2 \mathrm{Li} 2), \mathrm{X}(\mathrm{Cu} 4)] \backslash 1 @$

\section{$\operatorname{Ag}_{6} \operatorname{Li}_{2} C_{2 v}$ isomer:}

$1 \backslash 1 \backslash$ GINC-UGA-PVRS-

PC27PVRS \FOpt\RPW91PW91\LANL2DZ\Ag6Li2।WANNERE\22-M ar-2005\0\\# RPW91PW91/LANL2DZ OPT FREQ=NORAMAN POP=NONE NAME$=W A N N E R E \backslash$

$\backslash \mathrm{Ag} 6 \mathrm{Li} 2 \mathrm{C} 2 \mathrm{~h}$ symmetry $\backslash 0,1 \backslash \mathrm{Ag}, 0.0413107696,1.402446067,-1.463566264 \backslash \mathrm{Ag}$, 0.0413107696,-1.402446067,-1.463566264\Ag,0.0413107696,-1.402446067,1. $463566264 \backslash \mathrm{Ag}, 0.0413107696,1.402446067,1.463566264 \backslash \mathrm{Ag},-1.9433135769,0$. , $0.1 \mathrm{Ag}, 2.0210872625,0 ., 0.1 \mathrm{Li},-1.9036313192,2.6634819405,0.1 \mathrm{Li},-1.903631$ 3192,-2.6634819405,0.1IVersion=x86-Linux-G98RevA.5\HF=-890.0553922\RMS $\mathrm{D}=9.596 \mathrm{e}-09 \backslash \mathrm{RMSF}=8.827 \mathrm{e}-05 \backslash \mathrm{PG}=\mathrm{C} 02 \mathrm{~V}[\mathrm{C} 2(\mathrm{Ag} 1 \mathrm{Ag} 1), \mathrm{SGV}(\mathrm{Li} 2), \mathrm{X}(\mathrm{Ag} 4)] \backslash @$

\section{$\operatorname{Ag}_{6} \operatorname{Li}_{2} C_{2 h}$ isomer:}

$1 \backslash 1 \backslash$ GINC-UGA-PVRS-

PC31PVRSIFOpt\RPW91PW91 \LANL2DZ\Ag6Li2\WANNERE\24-N ov-2004l0\॥ RPW91PW91/LANL2DZ OPT FREQ=NORAMAN POP=NONE NAME $=$ WANNERE

SCFCYC=300\\Ag6Li2 C2h symmetry\\0,1\Ag,-0.9264907944,-1.4722152754,1. 0443595269\Ag,-0.9264907944,1.4722152754,1.0443595269\Ag,0.9264907944, $-1.4722152754,-1.0443595269 \backslash \mathrm{Ag}, 0.9264907944,1.4722152754,-1.0443595269$ $\backslash \mathrm{Ag}, 1.6017847818,0 ., 1.2293856902 \backslash \mathrm{Ag},-1.6017847818,0 .,-1.2293856902 \backslash \mathrm{Li}$, $0.2343961273,0 .,-3.164801444 \backslash \mathrm{Li},-0.2343961273,0 ., 3.164801444 \backslash \backslash V e r s i o n=$ $\mathrm{x} 86-\mathrm{Linux}-\mathrm{G} 98 \mathrm{RevA} .5 \backslash \mathrm{HF}=-890.051096 \backslash \mathrm{RMSD}=7.957 \mathrm{e}-09 \backslash \mathrm{RMSF}=1.166 \mathrm{e}-04 \backslash \mathrm{PG}=\mathrm{C} 0$ 2H [SGH(Ag2Li2),X(Ag4)]\@

\section{$\mathrm{Au}_{6} \mathrm{Li}_{2} C_{2 v}$ isomer:}

$1 \backslash 1 \backslash$ GINC-UGA-PVRS-

PC27PVRS $\backslash F O p t \backslash R P W 91 P W 91 \backslash L A N L 2 D Z \backslash A u 6 L i 2 / W A N N E R E \backslash 04-M$ ar-2005\0\॥ RPW91PW91/LANL2DZ OPT FREQ=NORAMAN POP=NONE NAME $=$ WANNERE $\backslash$

$\backslash$ Au6Li2 C2v symmetry $\backslash 0,1 \backslash A u,-0.0311273124,-1.4619899632,1.422536816 \backslash \mathrm{A}$ u,-0.0311273124,-1.4619899632,-1.422536816\Au,-0.0311273124,1.46198996 $32,-1.422536816 \backslash \mathrm{Au},-0.0311273124,1.4619899632,1.422536816 \backslash \mathrm{Au}, 2.0030378$ 
706,0.,0.\Au,-2.0161692098,0.,0.\Li,1.8122677536,0.,2.6485318736\Li,1. $8122677536,0 .,-2.648531873611$ Version $=x 86-$ Linux-G98RevA.5\HF=-828.37001 $68 \backslash \mathrm{RMSD}=9.995 \mathrm{e}-09 \backslash \mathrm{RMSF}=3.223 \mathrm{e}-05 \backslash \mathrm{PG}=\mathrm{C} 02 \mathrm{~V}[\mathrm{C} 2(\mathrm{Au} 1 \mathrm{Au} 1), \mathrm{SGV}(\mathrm{Li} 2), \mathrm{X}(\mathrm{Au} 4)] \backslash$ \@

\section{$\mathrm{Au}_{6} \mathrm{Li}_{2} C_{2 h}$ isomer:}

$1 \backslash 1 \backslash$ GINC-UGA-PVRS-

PC30PVRS\FOpt\RPW91PW91 \LANL2DZ\Au6Li2\WANNERE\24-N ov-2004\0\॥ RPW91PW91/LANL2DZ OPT FREQ=NORAMAN POP=NONE NAME $=W A N N E R E$

SCFCYC=300\\Au6Li2 C2h symmetry\10,1\Au,-0.9319822962,-1.4697802411,1. 0637727604\Au,-0.9319822962,1.4697802411,1.0637727604\Au,0.9319822962, -1.4697802411,-1.0637727604\Au,0.9319822962,1.4697802411,-1.0637727604 $\backslash \mathrm{Au}, 1.603204194,0 ., 1.2520250513 \backslash \mathrm{Au},-1.603204194,0 .,-1.2520250513 \backslash \mathrm{Li}, 0$. $2384259979,0 .,-3.130767245 \backslash \mathrm{Li},-0.2384259979,0 ., 3.130767245 \backslash$ Version=x8 6-Linux-G98RevA.5\HF=-828.3700323\RMSD=9.039e-09\RMSF=6.574e-05 $\backslash \mathrm{PG}=\mathrm{C} 02$ H [SGH(Au2Li2),X(Au4)]川@ 